\title{
Le suivi des enfants en voyage et l'usage du portfolio
}

Quelques résultats

\section{Marc Derycke}

\section{OpenEdition}

\section{Journals}

Édition électronique

URL : http://journals.openedition.org/esp/126

DOI : $10.4000 /$ esp. 126

ISSN : 2532-0319

\section{Éditeur}

Centre d'Information sur l'Éducation Bilingue et Plurilingue

Édition imprimée

Date de publication : 1 juin 2014

Pagination : 25-38

ISSN : 1127-266X

\section{Référence électronique}

Marc Derycke, "Le suivi des enfants en voyage et l'usage du portfolio ", Éducation et sociétés plurilingues [En ligne], 36 | 2014, mis en ligne le 25 octobre 2014, consulté le 01 mai 2019. URL : http:// journals.openedition.org/esp/126; DOI : 10.4000/esp.126 


\section{LE SUIVI DES ENFANTS EN VOYAGE ET L'USAGE DU PORTFOLIO. QUELQUES RÉSULTATS}

\section{Marc DERYCKE}

La scolarizzazione dei bambini nomadi necessita un controllo al fine di assicurare la coerenza e la progressione degli apprendimenti. Dopo una sperimentazione in tre paesi europei, si può notare che il portfolio è il metodo più adatto per trasmettere una rappresentazione fedele e valida del livello dell'alunno. Inoltre fornisce un beneficio cognitivo (in quanto lo costringe alla riflessione per ottenere l'informazione), ma anche culturale e relazionale, poiché gli permette di prendere coscienza delle aspettative e dei codici della scuola. Esso favorisce anche il rapporto con il suo ambiente familiare e ciò contribuisce all'acculturazione. Ma questo strumento è utile per tutti i bambini (è stato concepito per sedentari). La difficoltà della sua introduzione si spiega col fatto che esso rimette in causa il modello di trasmissione scolastica, profondamente radicato: obbliga ad un capovolgimento della pratica dell'insegnamento e dell'organizzazione della classe. La sua applicazione deve quindi essere accompagnata dall'autorità gerarchica.

The schooling of Travellers' children requires follow-ups to make sure the teaching is gradual and coherent. Following experimentation in three European countries, it turns out the portfolio is best for transmitting a faithful and valid representation of the pupil's level. There are cognitive benefits too, because to be adequately filled in, the portfolio requires reflexivity, as well as cultural and relational benefits, because it allowes pupils to become aware of the codes and expectations of the school; it also enhances the relationship with their family environment. We point out that the tool is useful for all children (it was originally conceived for sedentary children). Introducing the portfolio only presents a difficulty because it challenges the deep-rooted academic model of transmission: it means changing teachers' habits and the organization of the class. Implementing it must therefore be accompanied by the hierarchy.

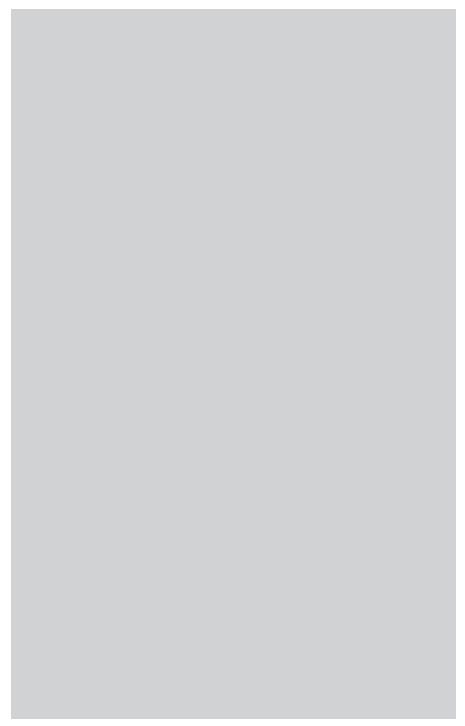

es travaux réalisés par l'équipe que j’ai dirigée sur le suivi pédagogique des enfants dits «du voyage» (1) ont duré sept ans (2). Leur démarrage et leur aboutissement n'auraient pas été possibles en France sans l'encouragement et l'aide des enseignants recevant ces enfants. L'événement décisif a été l'organisation de deux réunions de travail à Dijon, en 1993 et 1994, sous l'impulsion de Régis Alvis et de l'École des Voyageurs, située sur une aire de stationnement de la région. D’une part, le suivi pédagogique était mis au premier plan des préoccupations enseignantes, la recherche pouvait donc s'en emparer. De plus, les rencontres faites à cette occasion ont été le point de départ d'une coopération fructueuse avec des enseignants et des maîtres formateurs de CEFISEM puis CASNAV (3), ceux d'Ile de France particulièrement, mais aussi de Glermont- 
Le suivi des enfants en voyage et l'usage du portfolio. Quelques résultats

M. DeRYCKe
Ferrand. Les résultats sont maintenant accessibles (voir Références bibliographiques).

La présente contribution présentera donc l'expérience initiale et ses développements et signalera enfin ce qui me semble actuellement constituer les principaux résultats de ce travail, mais aussi ses limites.

Une expérimentation de deux types d'outils de conception contrastée a été réalisée auprès de 200 enseignants et stagiaires en fin de formation en Belgique et Suisse francophones ainsi qu'en France (4). Ces outils étaient de deux types: la grille «critériée», document d'évaluation classique, réalisé sur le modèle de celui que le Ministère avait proposé en 1991; et le «dossier d'apprentissage», ou portfolio (5), outil qualitatif «réinventé» par des institutrices d'école maternelle en divers lieux de France. Je l'ai découvert à Saint-Etienne en 1991, mis en œuvre depuis plus d'un an par une équipe de formatrices en maternelle composée notamment d'Edith Grasset et de Christine Segreto. Ensuite, j'en ai reçu un exemplaire appartenant à un enfant forain anglais, enfin Jacques Weiss (IRDP, Neuchâtel) m'a communiqué les travaux existants sur la question, essentiellement anglo-saxons, par le biais de Jean-François de Pietro. Le portfolio fait maintenant partie des outils novateurs officiellement recommandés dans les nouvelles méthodes d'évaluation individualisée; il l'est paradoxalement moins dans l'enseignement élémentaire et secondaire que dans la formation professionnelle des adultes, dont celle des enseignant(e)s et des infirmier(e)s. Ce «recueil cumulatif d'extraits choisis et commentés des travaux de l'élève» peut soutenir soit une démarche d'évaluation formative et formatrice, soit même être un support d'évaluation sommative, voire certificative (6), voir les pionniers en la matière (Simon \& Forgette-Giroux 1993).

L'expérimentation a montré sans équivoque la supériorité du portfolio. Elle cherchait en priorité à évaluer les informations évaluatives du livret de l'élève en fonction de deux variables: leur fidélité (les informations rendent-elles bien compte de sa performance, voire de ses compétences?), et leur validité (ces informations présentent-elles bien la performance ou les compétences de cet élève-là, et pas d'un autre?), afin de situer l'enfant et lui faire poursuivre ses apprentissages sans retard.

De manière synthétique, nous dirons que ces avantages tiennent au fait que, grâce aux traces commentées en principe par l'enfant, ceci de manière directe ou indirecte (dictée à l'adulte ou à un camarade), quel que soit le niveau de formation de l'enseignant et son expérience (je rappelle que l'expérimentation a eu lieu aussi avec des stagiaires), donc quel que soit l'enseignant, le jugement évaluatif sur une même perfor- 
Le suivi des enfants en voyage et l'usage du portfolio. Quelques résultats

M. DeRYcke mance était relativement homogène et concordant. Ces deux points sont très importants pour ce qui concerne le suivi: lors de l'expérimentation, les enseignants qui recevaient des livrets renseignés par plusieurs de leurs collègues, mais présentant chacun à leur manière la même performance de l'élève, portaient un jugement sensiblement identique; ceux-ci étaient donc homogènes. De plus, ce jugement est valide: en effet, quand ces enseignants récepteurs (c'est-à-dire en position de recevoir l'enfant dès son arrivée) étaient mis dans un second temps en situation de vérifier la performance «réelle» (enregistrée en vidéo) de l'élève, telle qu'elle avait été encodée par les destinateurs dans le livret (ceci à l'aide d'une sélection commentée des traces), ils ne trouvaient pas de différence sensible avec la représentation qu'ils s'étaient faite dans un premier temps lors de la lecture du portfolio. Il y a donc, pour un même enseignant, concordance entre la représentation construite à la lecture du livret et le jugement direct de la performance qui a servi à l'encodage.

L'expérimentation a été particulièrement concluante avec l'enfant en difficulté; or, c'est avec ce type d'enfant que la grille critériée présentait des informations souvent contradictoires, voire même ininterprétables, et quand les interprétations étaient homogènes, il est apparu qu'elles surévaluaient l'élève, la marge d'erreur sur la détermination du niveau allant même jusqu'à près d'une année scolaire. En effet, d'une part, des enseignants ne veulent pas en donner une image négative; de l'autre, renseignant les items l'un après l'autre, certains ne se mettent pas à la place du récepteur pour vérifier si l'image globale offerte par l'ensemble des items est cohérente et conforme à leur perception du niveau de l'élève; enfin, ces deux travers peuvent se cumuler. Ces défauts sont cruciaux. En effet, d'une part, les enfants itinérants sont très souvent perçus comme étant en échec scolaire, non socialisés, etc., de l'autre, s'ils possèdent souvent un niveau scolaire au-dessous du niveau moyen de la classe qui les accueille, ils ne sont pas dépourvus, bien au contraire, de compétences et de savoirs acquis. Or l'enseignant risque de se fier à ses préjugés en les considérant «en échec», il faut donc apporter des informations précises pour qu'il/elle tienne compte de la spécificité de l'élève, afin de lui proposer des activités appropriées tout en le mettant en confiance. La fidélité et la validité des informations sont donc indispensables: proposer des tâches largement au-dessus des compétences de l'enfant, c'est le mettre en difficulté alors qu'il est déjà insécurisé par un environnement nouveau et des codes scolaires qu'il ne maîtrise souvent pas ou peu. L'avantage du portfolio vient du fait qu'il permet un ajustement à l'élève et inversement, celui-ci étant mis en situation de pratiquer consciemment le code scolaire en ce qu'il l'élabore en se positionnant par rapport aux 
Le suivi des enfants en voyage et l'usage du portfolio. Quelques résultats

M. DERYCKE

\section{REMARQUE IMPORTANTE:}

\section{QUELS OBJECTIFS, QUELS PRINCIPES?}

attentes de l'enseignant - pourquoi je fais ceci, cela, dans quel but, etc. qu'il peut donc en parler; bref, qu'il se l'approprie, ce document devenant bien le sien, au point qu'il lui soit difficile de s'en séparer.

Enfin, le portfolio est peu sensible au jugement de l'enseignant précédent. La présence des traces permet de corriger un commentaire fait par le prédécesseur avec lequel l'enseignant récepteur peut alors nourrir un désaccord: si ces traces sont suffisantes, il peut se faire son propre jugement en fonction de sa propre pratique et de ses convictions pédagogiques. De plus, le portfolio, s’il est bien organisé, est rapide d'interprétation. Enfin, la présence de traces permet de ne pas faire reposer l'interaction uniquement sur du déclaratif et, partant, sur l'usage de la langue, lequel dresse des obstacles qui peuvent handicaper la communication (niveaux de langue socialement catégorisés, voire contact exolingue).

Parallèlement à l'expérimentation, nous avons conçu par étapes un portfolio qui tenait compte de ces résultats. Celui-ci demande un temps suffisant pour que l'enfant se l'approprie grâce aux conditions que l'enseignant a créées dans la classe; dans les cas où l'enfant reste trop peu de temps dans l'école pour que lui et l'enseignant s'en emparent, nos collègues anglais ont conçu à l'époque une «carte d'objectifs» (Target card), respectant l'esprit du portfolio, sur laquelle l'enfant s'engage. Ainsi, il pourra lui-même montrer dans ses cahiers ce qui correspond à sa réalisation et en expliquer éventuellement les étapes et les particularités.

Les résultats que peut proposer une recherche scientifique sont toujours en décalage avec les attentes des praticiens. En effet, une recherche isole un très petit nombre de variables pour les soumettre à l'examen. Ces résultats sont donc parcellaires au regard de la complexité des variables que rencontre l'enseignant, lesquelles sont loin d'être toutes identifiées. Aussi les propositions d'outil qui ont été faites à partir de nos conclusions gardaient-elles un caractère largement exploratoire: c'est l'appropriation par les enseignants qui allait permettre de les remanier, à quoi Andrée Chastel (2012) a très largement contribué.

L'objectif prioritaire auquel le suivi pédagogique doit contribuer est moins de faire un état des apprentissages de l'élève (objectif tactique) que de l'y enrôler et pour cela, donner du sens à ces activités et plus généralement à la fréquentation de l'école (objectif stratégique). La mise en œuvre pour atteindre cet objectif repose sur la mobilisation des pratiques appropriées de retour réflexif, la tenue du portfolio nécessitant ce retour sur les activités afin de les analyser, de repérer les 
Le suivi des enfants en voyage et l'usage du portfolio. Quelques résultats

M. DeRYcke difficultés et les aides nécessaires, de rendre compte des découvertes... Le principe est bien que l'enfant saisisse le sens de sa présence dans l'établissement scolaire et par là, celui des apprentissages, de manière à ce qu'il comprenne les critères et modalités de la mise au travail, mais aussi les critères de jugement applicables à ses productions. $\mathrm{Ce}$ qui peut se décliner ainsi:

- qu'attend-on de moi?

- qu'ai-je fait, qu'aurai-je dû faire pour correspondre à cette attente?

- comment procéder dans la prochaine activité pour la mener à bien?

Ceci nécessite notamment pour l'élève d'être capable d'identifier les objectifs et compétences à travailler, d’identifier les domaines disciplinaires auxquels appartient l'activité, puis de choisir les procédures adéquates et de planifier son action, la contrôler, etc. Ces éléments s'inspirent à la fois de l'évaluation formatrice et de pratiques dites «métacognitives», soit une forme de réflexivité. Cependant, on observe que leur mise en œuvre donne souvent lieu à une surcharge stérile de travail. Les opérations menant à l'exécution de la tâche puis à son évaluation sont décomposées académiquement et entrent dans un parcours rigide d'instructions formelles, comme si les élèves étaient ignorants en tout et n'étaient pas capables d'emprunter des passerelles et des raccourcis. Précisons que le sens dont je parle ici appartient à la logique de l'action. Il ne surgit que dans la simplicité que confère la focalisation sur l'enchaînement temporel des seules priorités où la régulation consciente est indispensable. Ceci n'est réalisable que lorsqu'est proposé un cadre balisé par des principes peu nombreux mais éprouvés, ainsi chacun gagne en autonomie, peut s'autoriser à tâtonner avant de tirer globalement les leçons de la mise en œuvre. Ceci est à l'opposé des procédures académiques dont la grille critériée est le reflet, lesquelles reposent sur l'exhaustivité des critères d'une tâche qui conduisent à son atomisation.

Mais un dispositif de suivi pédagogique répondant à ces principes et partageant ces objectifs ne peut être mis en place pour quelques enfants «spéciaux», par quelques enseignants à qui on demanderait alors d'être «spécialisés». Non: l'exigence qui vise le sens concerne tout enfant, elle est à mettre en œuvre par tout enseignant.

Le dispositif que nous préconisons concerne en effet tous les enfants et apprenants, à tous les niweaux, même si sa contextualisation est toujours particulière. C'est à cette condition que le droit peut être respecté: tout enfant en voyage (pour quelque raison que ce soit) est susceptible d'être accueilli dans toute école de la République, son suivi, comportant certes quelques aménagements fonctionnels, n'est qu'une extension du dispositif d'activité de retour réflexif et de suivi, dispositif créé pour tous. C'est pourquoi ce qui est une exigence indispensable pour 
Le suivi des enfants en voyage et l'usage du portfolio. Quelques résultats

M. DeRYcke

\section{MISE EN (EUVRE ET MISE EN COHÉRENCE DES PRATIQUES QUOTIDIENNES}

favoriser la scolarisation des enfants mobiles l'est aussi pour les autres. Lorsque les classes auront adopté ce dispositif pour les élèves sédentaires, l'enfant itinérant qui s'y présentera sera enrôlé naturellement dans cette logique par ses pairs et l'enseignant; sa prise en charge individuelle en sera allégée d'autant. La mise en œuvre d'un dispositif de cette nature concerne donc aussi tous les enseignants.

Objectif qui pourrait être jugé secondaire par l'administration, voire par les enseignants, le portfolio est aisément lisible par tous les parents, et est peu sensible à leur degré d’instruction. Des extraits du travail luimême commentés par l'enfant sont donnés à voir au lieu d'être désignés par un code ou un jargon de professionnels. Dès lors, l'enfant peut apporter des explications en ce qu'il a été actif lors de la sélection et des commentaires. Cette lisibilité contribue au sens en ce qu'elle le rend accessible à ceux qui peuvent s'en faire porteur pour le répercuter sur la famille: pour que l'école et les apprentissages aient une signification, il n'est pas toujours inutile que celle-ci soit partagée par les membres de son entourage, ce qui légitime en retour le statut d'élève que l'enfant acquiert.

Nous l'avons signalé ci-dessus, la mise en cohérence des pratiques quotidiennes de l'enseignant est la condition préalable à la mise en œuvre d'un document de suivi pédagogique conçu selon nos principes. L'objectif est de faire intégrer par l'enseignant une conception de la démarche évaluative telle qu'elle soit le moyen de pilotage des apprentissages, ce qui situe cette démarche au centre des apprentissages eux-mêmes.

Les régulant et les orientant, cette démarche appartient aux séquences d'apprentissages; celles-ci ne peuvent se produire en dehors de l'élève, il doit donc y être associé. Certaines conceptions préconisent de l'associer à tout. Certes, l'élève doit participer au processus, mais il s'agit de repérer quelle est la responsabilité de l'enseignant, sa place, et en face, celle spécifique de l'élève et partant, la nature de sa collaboration. Sa visée est d'alléger l'activité cognitive de l'élève en l'orientant vers le but et pour cela ne retenir que ce qui est à la fois indispensable et éclairant pour lui dans sa «zone de proche développement» (7) afin de lui permettre d'assurer ce qui relève de sa responsabilité: l'élève doit pouvoir assumer pleinement son rôle d'apprenant en fonction de son niveau de développement et pour cela utiliser son temps et son énergie de manière efficace afin d'atteindre avec succès les objectifs raisonnables qui lui sont assignés.

Certes, tous les enseignants proposent à leurs élèves des repères, tous les incitent à réfléchir à ce qu’ils font, à tirer parti de leurs erreurs. En revanche, nombre d'enseignants disjoignent ces pratiques qui, dès lors, 
Le suivi des enfants en voyage et l'usage du portfolio. Quelques résultats

M. DeRYCKE

\section{LISIBILITÉ}

L'ÉVALUATION COMME PILOTAGE DE L'ACTIVITÉ

GESTION DU TRAVAIL PERSONNEL perdent leur sens et semblent répétitives et abrutissantes aux élèves en ce qu'elles ne s'intègrent que durant les moments qui y sont consacrés en les soumettant à une visée globale: la réussite de telle activité nécessite un ensemble cohérent et économe dans lequel chaque segment prend place pour contribuer aux objectifs signalés ci-dessus,

- soit l'observation/construction de repères collectifs pertinents pour l'action, ceci en associant dialectiquement groupe, grand et petit, et individu; ce qui ne peut s'envisager sans leur validation après-coup,

- soit les retours réflexifs sur telle activité, tel travail et moment de vie de classe où ces repères étaient susceptibles de s’appliquer.

À cette fin, les contenus d'enseignement doivent être rendus lisibles pour tous par identification de leur objet (quoi? normes, compétences, savoirs disciplinaires...), c'est pourquoi les conditions (quand, où, comment?) de cette identification doivent être mûries et organisées. Par exemple, l'enseignant et, partant, les élèves, devraient avoir une représentation claire de ce que sont les normes scolaires appliquées, les compétences à travailler, les savoirs organisés en disciplines; où, quand et comment, voire avec qui ces objets sont mobilisés. Ceci demande de procéder par des affichages qui «parlent» aux élèves et qui leur permettent à tout moment de se situer dans les apprentissages; car il s'agit à propos de toute tâche de saisir en quoi l'activité est bien celle de l'école. Une fois ce cadrage intégré, il ne sera plus nécessaire d'y accorder une priorité; un rappel occasionnel suffira.

L'activité réflexive, dans une perspective formative, prend pour objet ceux qui sont rendus lisibles; l'enseignant peut (se) demander quels sont les objets à soumettre à l'analyse critique (quoi? en principe au sein d'une seule discipline, pour ne pas alourdir la démarche), ainsi que les conditions de réalisation de cette analyse (quand, où, comment?).

C'est dans ce temps d'appropriation que la sélection des traces d'activités rendant compte du processus réflexif et leur commentaire en interaction avec un tiers, prennent toute leur signification et apparaissent fonctionnels. Un document qui les recueille apparait alors le nécessaire aboutissement de ces pratiques convergentes: un cahier, un classeur. Le portfolio, permettant d'assurer le suivi pédagogique, est le document qui permet d'objectiver ce processus. Son usage avec les enfants en voyage demande seulement quelques précautions afin de tenir compte des contraintes du voyage et de celles du passage de mains en mains: légèreté (seuls les feuillets récents sont conservés, les autres sont gardés dans un classeur qui reste dans l'école principalement fréquen- 
Le suivi des enfants en voyage et l'usage du portfolio. Quelques résultats

M. DeRYCKe

\section{REMARQUES}

ACCOMPAGNEMENT ADMINISTRATIF: LE «GROUPE DE PILOTAGE» tée lorsque c'est le cas), rusticité (le document doit résister aux intempéries, aux benjamins...), comporter un mode d'emploi et fournir des informations administratives... Si le moment n'est pas venu pour son introduction (soit défaut de structure administrative d'accompagnement, soit l'enseignant et sa classe, ou l'élève, ne sont pas prêts), la «Carte d'objectifs» peut suffire, qui se bornera à mentionner les seules priorités, sans comporter les matériaux qui ont alimenté la dimension réflexive.

La mise en cohérence de ces segments doit différencier, d'une part, ce qui revient au rituel qui structure le groupe et les procédures, fournissant des repères externes pour être internalisés par l'individu (procès de la répétition), et d'autre part, ce qu'il soutient, essentiel, et qui le dépasse, où l'élève a à jouer sa partie: le procès individuel de conquête par lequel l'élève investit l'inconnu en s'appuyant sur le connu, les aides (étayage) fournies par l'environnement et les interactions (procès d'élaboration du nouveau). S'il ne sert pas à accompagner souplement ce second procès, le cadre ci-dessus peut fort bien l'annihiler et alimenter un académisme procédurier bien présent dans l'idéologie de l'appareil scolaire; il s'agit donc d'inciter les enseignants à s'en émanciper en comptant sur leur ingéniosité appliquée in situ.

Par ailleurs, ce double procès engage l'apprentissage progressif par l'élève de la saisie de l'attente que nourrit l'enseignant et la prise en compte de son altérité, laquelle se traduit dans l'évaluation certificative et s'éprouve, par delà la rationalisation des pratiques et leur explicitation, au travers d'un reste irréductible d'arbitraire: soumettre une production à un évaluateur, présuppose d'assumer cette altérité.

Le suivi pédagogique des enfants en voyage ne peut être laissé à l'initiative individuelle de tel ou tel enseignant motivé, de tel ou tel parent ou de quelques inspecteurs sensibilisés. Comment comprendrait-on que l'Administration ne s'implique pas dans la prise en charge de son organisation et de sa régulation à l'échelle du territoire? Encore lui faut-il un outil pour cela.

L'expérience tant internationale que nationale en la matière montre que l'outil le plus efficace en ce domaine est celui que nous avons baptisé «Groupe de pilotage» (Derycke, Macret et al. 2000). Cette institution est rendue nécessaire par la dispersion dans le temps et dans l'espace de la fréquentation scolaire de ces publics, par l'émiettement et la nouveauté des problèmes qu'elle pose. La centralisation de ses missions permet de donner cohérence et économie dans la gestion de ce qui, si non, n'est pris en charge que de manière partielle, hétéroclite et avec un coût important; elle doit se trouver sous l'autorité du supé- 
Le suivi des enfants en voyage et l'usage du portfolio. Quelques résultats

M. DeRYCKe
CONCLUSIONS, PERSPECTIVES rieur académique). Devraient lui incomber:

- l'initiation du suivi pédagogique, avec son volet de formation préalable et d'accompagnement pédagogique des enseignants au cours de la mise en place du dispositif,

- la mise en relation des écoles et établissements qui reçoivent ces publics; particulièrement leur répartition, adoptée à l'étranger (Royaume-Uni, Pays-Bas, depuis plus de dix ans, dans certains Länder d'Allemagne), en un double réseau:

- celui des «écoles d'hiven», soit celles où les enfants sont scolarisés plus longtemps, où les nouveaux outils et les nouvelles pratiques peuvent donc être initiés dans la durée, de manière à ce que l'enfant et les parents en comprennent le fonctionnement et puissent le répercuter ailleurs,

- celui des «écoles d'été», quand la durée de fréquentation sera plus courte; c'est leur fréquentation qui réclame particulièrement l'existence d'un dispositif de suivi efficace, ceci bien que ces dernières soient les plus fragiles pour l'assurer. Elles doivent donc être l'objet de mesures d'accompagnement attentif sous peine de ruiner les efforts faits par les écoles d'hiver.

- le suivi administratif et la logistique du dispositif (conception, édition, distribution du document de suivi pédagogique (8), contrôle de leur usage et de leur transmission d'une école à l'autre),

- coordination avec les différents partenaires, au besoin au travers d'un «Groupe partenaire»: centres de formation, municipalités, parents, représentants des différents ministères, etc.

Les résultats partiels dont il a été fait état ici reposent sur un choix lié à la situation du début des années 2000; elles sont toujours d'actualité, l'avenir montrera sans doute que d'autres auraient pu être faits. Rappelons que le suivi pédagogique n'a pas constitué un objet de recherche proprement dite, sauf celles que nous avons menées, aussi une réserve s'impose: nous avons privilégié une approche, d'autres mériteraient sans doute d'être développées.

L'essentiel en cette affaire est moins une prise en compte mécaniste des résultats de ces recherches que le repérage des principes de mise en œuvre qu'il s'agit de prendre en compte afin d'orienter la pratique. Mais ceci ne peut se faire hors de tout accompagnement formatif de la part du conseiller pédagogique, mandaté par l'instance administrative (Groupe de pilotage). On a vu que la mise en place volontariste d'un document de suivi, en contradiction avec ces principes, menait à l'abandon de l'outil parce que celui-ci paraissait plus lourd qu'utile à l'usage. À ceci deux raisons: pour l'essentiel, son bénéfice pédagogique 
Le suivi des enfants en voyage et l'usage du portfolio. Quelques résultats

M. DeRYcke immédiat dans la gestion des activités d'apprentissage en classe n'aura pas été perçu s'il n'est rappelé par le coordonateur ou le conseiller, ce qui a pour conséquence de priver de sens sa mise en œuvre pratique. Cependant, si on attend que la situation interne soit mûre pour introduire le portfolio, il ne le sera jamais. En effet, penser que des pratiques cohérentes qui mettent en œuvre ces principes permettraient à terme sa mise en place efficace est un leurre s'il n'y a pas intervention de l'extérieur: la pression du quotidien est toujours la plus forte pour remettre au lendemain l'introduction d'un outil qui contraint à des modifications importantes des représentations traditionnelles du métier. En fait, il doit être imposé de manière anticipée par l'autorité administrative pour que les problèmes se posent concrètement, ceci en développant simultanément une réflexion sur les pratiques, le tout grâce à un accompagnement pédagogique adéquat. C'est pourquoi la mise en place du portfolio et des pratiques de suivi doit être coordonnée par un groupe de pilotage au niveau du département sinon audelà.

Considérant, avec le recul d'une dizaine d'années, le peu d’impact qu'a eu la mise en œuvre du portfolio dans les classes et particulièrement auprès des enfants en voyage, ceci alors qu'il est maintenant vivement encouragé, voire imposé, dans les formations professionnelles qui se veulent innovantes, montre sans doute:

- L'absence de groupe de pilotage, à tout le moins de directives et de suivi sous l'autorité d'une instance légitime convaincue que l'usage de cet outil doit être lié à la mise en œuvre d'une différentiation pédagogique conséquente, au bénéfice de tous les élèves;

- Sa perméabilité aux résistances des enseignants, au nom de la surcharge de travail que cela semble leur imposer, raison particulièrement invoquée par ceux qui reçoivent des enfants du voyage, réagissant dans l'urgence face à des problèmes matériels et sociaux, sans considérer que les apprentissages doivent être menés de front, sinon avoir la priorité pour que la fréquentation scolaire prenne sens;

- L'absence de mise en œuvre guidée pas à pas en prenant en compte les obstacles rencontrés par les enseignants, afin de les résoudre de manière formative en tutorat sous forme de cas;

- La présence de freins internes, lesquels proviennent de modèles bien enracinés chez les pédagogues: prégnance insoupçonnée du modèle transmissif en dépit des orientations de la formation «professionnalisante», méfiance face aux innovations venant de l'université, poids de la dimension relationnelle: par exemple, telle innovation n’a été appliquée que dès lors que s'est établie une relation forte avec son promoteur universitaire...

- Que la recommandation légitime du portfolio semble en définitive se 
Le suivi des enfants en voyage et l'usage du portfolio. Quelques résultats

M. DeRYcke

ANNEXES

CONSTRUIRE SON LANGAGE INITIATION

À L'ÉCRIT

\section{GUIDE:}

réduire à un affichage dans une stratégie de communication d'institutions voulant se présenter sous le jour de l'innovation. Ainsi, il est recommandé voire imposé là où c'est le plus facile et, peut-être, le moins utile, comme c'est le cas ici ou là dans la formation professionnelle, celle des enseignants, celle des infirmier(e)s, alors que ces apprenants sont déjà imprégnés de culture scolaire et ont intériorisé les pratiques réflexives. En revanche, ce n'est guère le cas là où l'enjeu est majeur: en début de scolarisation. C'est sur ce segment qu'il faut agir!

Mais comment? Qui le fera?

1) Quels sont les supports, thèmes, activités qui vous semblent pouvoir mettre l'enfant à l'aise lors du premier contact

2) Donnez les indications qui vous semblent utiles dans l'une et l'autre colonne (seuls les éléments nouveaux sont signalés). Glissez au verso et dans les pochettes suivantes des exemples de réalisation ou d'exercice choisis et commentés avec l'élève, ou à défaut, son cahier.

\begin{tabular}{|l|l|l|l|}
\hline $\begin{array}{l}\text { COMPÉTENCES EN COURS } \\
\text { D'ACQUISITION (dater) }\end{array}$ & $\begin{array}{l}\text { AcQuIS } \\
\text { (dater) }\end{array}$ & $\begin{array}{l}\text { À FAIRE } \\
\text { (activités possibles) }\end{array}$ & $\begin{array}{l}\text { FAIT } \\
\text { exercices, } \\
\text { manuels (dater) }\end{array}$ \\
\hline
\end{tabular}

* Écoute-t-il les autres, les comprend-il, répond-il de manière pertinente, participe-t-il à des échanges, et comment?

* Mémorise-t-il et est-il capable de réciter un texte court?

* Comment désigne-t-il une action, des objets familiers, des sentiments? Mémorise-t-il et utilise-t-il dans son contexte un vocabulaire précis?

Est-il capable d'appréhender grâce au contexte les différents sens d'un mot? Connaît-il quelques homonymes et synonymes? Connait-il le lexique nécessaire à la compréhension de consignes simples?

* Emploie-t-il différents registres de langue en fonction des situations?

* Organise-t-il logiquement son propos?

* Sait-il faire partager un événement ou un récit préalablement inconnu des personnes présentes? Sait-il compléter des phrases à trou?

* Comment se représente-t-il ce qu'on doit faire pour lire? Comment se représente-t-il ce que l'écrit codifie? Pour quelles raisons pense-t-il qu'il faut apprendre à lire?

* Sait-il de quoi se compose la phrase, de quoi se compose le mot, etc.? 
N.B. Ce guide est placé ici pour information. Dans le document en usage, les Guides d'évaluation disciplinaire ne se trouvent pas dans la partie à renseigner, mais rassemblés en fin de livret.

\section{MA CARTE D’OBJECTIFS (FORMAT A4 PLIÉ EN TROIS VOLETS)}

\begin{tabular}{|c|c|c|}
\hline |Recto| & Ce que je sais faire...... & Liste des écoles fréquentées..... \\
\hline MA CARTE DOBJECTIFS & Ce que j’aime faire à la maison,.... & Liste des livres que j'ai lus.... \\
\hline Nom... & Ce que j'aime faire à l'école.... & \\
\hline \multicolumn{3}{|l|}{ [Verso] } \\
\hline Date & Date & Date \\
\hline Mes objectifs sont.... & Mes objectifs sont... & Mes objectifs sont.... \\
\hline Réussi! & Réussi! & Réussi! \\
\hline 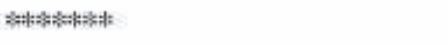 & 使: & 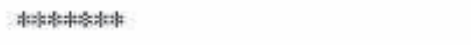 \\
\hline Date & Date & Date \\
\hline Mes objectifs sont.... & Mes objectifs sont.... & Mes objectifs sont.... \\
\hline Réussi! & Réussi! & Réussi! \\
\hline
\end{tabular}

\section{NOTES}

(1) La question que nous tentions de résoudre était celle de la rupture scolaire: quitter telle école pour en intégrer une autre après un laps de temps plus ou moins court, ou long, éventuellement revenir dans le même établissement. Cette rupture est surtout engendrée par le voyage, mais ceci peut valoir aussi pour d'autres causes. Les populations concernées peuvent être des Tsiganes, mais certaines se sédentarisent ou se sont sédentarisées, parmi celles-ci, certaines reprennent le voyage ou le reprendront. Le voyage est aussi justifié par des causes économiques: les travailleurs saisonniers, les forains ou les circaciens ne sont pas tous tsiganes... d'autant que la précarité économique amène des sédentaires à adopter un mode de vie proche.

(2) European Federation for the Education of the Children of the Occupational Travellers, actuellement dissoute. Organisation non gouvernementale de droit belge créée en 1988 et mandatée par la Commission des Communautés Européennes pour favoriser la scolarisation des enfants de travailleurs itinérants pour raison professionnelle en relation avec la Résolution du Conseil et des Ministres de l'Éducation réunis au sein du Conseil du 22 mai 1989 visant le soutien à l'éducation de ces publics; une Résolution parallèle concernait l'éducation des Tsiganes et Voyageurs, elle a été mise en œuvre par le Centre de Recherches Tsiganes (CRT, Université Paris 5). L'équipe de 
Le suivi des enfants en voyage et l'usage du portfolio. Quelques résultats

M. DERYCKE chercheurs était constituée de A.-C. Silva, B. Heijs, F. Tollmien, respectivement des institutions suivantes: Institut Supérieur de Psychologie appliquée, Lisbonne; Seminarium voor Orthopedagogiek (Pays-Bas); Université de Essen (Allemagne).

(3) CEFISEM: Centres de formation et d'information pour la scolarisation des enfants de migrants, crées en 1975; CASNAV: Centre Académique pour la Scolarisation des enfants Allophones Nouvellement Arrivés et des enfants issus de familles itinérantes et de Voyageurs, crée en 2002, chargé de l'accompagnement de la scolarisation d'une part, des élèves nouvellement arrivés en France sans maîtrise suffisante de la langue française ou des apprentissages scolaires et d'autre part, des enfants du voyage.

(4) Les résultats sont accessibles dans Derycke (2000b) pour un examen exhaustif des réponses lors d'une séance d'expérimentation.

(5) Le portfolio est une collection de travaux ou de productions réalisée dans un but précis. Mais c'est plus qu'une simple collection! En matière d'apprentissage, le but précis dont il est question est celui de permettre à chaque élève de rendre compte de ses réalisations, accompagnées de l'évaluation qu’il en fait (autoévaluation), et de rendre compte de son progrès. Il ne s'agit pas d'un simple passage d'un instant à un autre mais du progrès réalisé au regard d'une habileté particulière ou d'une compétence. Il faudrait peut-être ajouter: autant de compétences, autant de portfolios!

(6) L'évaluation formative vise l'acquisition par l'élève des critères attendus par le système éducatif, et partant, ceux de l'enseignant; elle repose sur la prise en compte par l'élève des critères appliqués par l'enseignant afin qu'il y conforme son travail. Ces critères lui sont donc préalablement communiqués, le préparant ainsi à une auto-évaluation ajustée à celle de l'enseignant. L'évaluation sommative sert à faire l'état des savoirs acquis; l'évaluation certificative est celle qui sanctionne une épreuve visant l'obtention d'un grade.

(7) Introduit par le psychologue soviétique Lev Vygotski bien avant la seconde guerre mondiale, ce concept qui fait l'objet d'une abondante littérature désigne la zone dans laquelle l'apprenant est capable de résoudre des problèmes non pas en autonomie, mais avec une aide; un exemple paradigmatique est fourni par la mère accompagnant son petit enfant à terminer avec succès un puzzle: la mère décharge la mémoire de travail de l'enfant des tâches secondaires, l'aide à se recentrer et peut éventuellement lui suggérer des voies de solution.

(8) La hiérarchie peut officiellement promouvoir tel outil, celui que nous préconisons par exemple, ou un autre, ou laisser le choix... 


\section{Le suivi des enfants en voyage et l'usage du portfolio. Quelques résultats \\ M. DeRYCKE \\ RÉFÉRENCES ET BIBLIOGRAPHIE INDICATIVE}

CHASTEL A. 2013. Comment améliorer la scolarisation des enfants itinérants? Éducation et Sociétés Plurilingues n 34 (juin): 43-54.

DERYCKE, M. 2000a. Le suivi pédagogique: des usages aux définitions, Revue française de pédagogie, n 132, Evaluation, suivi pédagogique et portfolio, Paris, INRP, pp .5-10.

—. 2000b. La grille critériée et le portfolio à l'épreuve du suivi pédagogique, Revue française de pédagogie $\mathrm{n}^{\circ} 132$, Évaluation, suivi pédagogique et portfolio, Paris, INRP, pp. 23-32.

- 2000c. École pour la majorité / écoles pour les minorités / École pour tous - éléments pour un débat, Revue du C.R.E., n¹8, Le suivi pédagogique des enfants en voyage, Saint-Etienne, Université J. Monnet, pp. 147-164.

-, MACRET J.-C. et al. 2000. Orientations pour le suivi pédagogique des enfants itinérants, Confluence(s) n 11, pp. 50-63.

HALLIDAY, T. 2000. Évaluation et suivi des enfants d'itinérants dans l'enseignement primaire: état des lieux du système des dossiers pédagogiques au Royaume-Uni, Revue du C.R.E., n 18, pp. 79-97.

OUZOULIAS A. 2000. Penser la différenciation et la continuité en s'appuyant sur la distinction entre apprentissage et développement, Confluence(s), n 11, pp. 31-49.

SILVA A.-C., HEIJS B. 2000. Indices et procès de l'apprentissage en lecture: réflexions pour la guidance, Revue du C.R.E., n 18, pp. 23-65.

SIMON, M., FORGETTE-GIROUX, R. 1993. Vers une utilisation rationnelle du dossier d'apprentissage, Mesure et évaluation en éducation, vol. 16, n 3-4, pp. 25-33.

WEISS J. 2000. Le portfolio, instrument de légitimation et de formation, Revue française de pédagogie, n¹32, Évaluation, suivi pédagogique et portfolio, Paris, INRP, pp. 11-22.

WILLIAMS P. 2000. Les enfants du voyage: scolarisation et suivi pédagogique, Confluence(s), n 11, pp. 14-17. 